Supporting Information

\title{
Highly Lithiophilic Cobalt Nitride Nanobrush as a Stable Host for High-Performance Lithium Metal Anodes
}

Meina Lei, ${ }^{\dagger}$ Jian-Gan Wang,,$^{* *}$ Lingbo Ren,,$^{\dagger}$ Ding Nan,,,$^{*}$ Chao Shen,${ }^{\dagger}$ Keyu Xie,,$^{\dagger}$ Xingrui Liü

† State Key Laboratory of Solidification Processing, Center for Nano Energy Materials, School of Materials Science and Engineering, Northwestern Polytechnical University and Shaanxi Joint Lab of Graphene (NPU), Xi'an 710072, China;

\$ School of Materials Science and Engineering, Inner Mongolia University of Technology, Aimin Street 49, Hohhot 010051, China

Corresponding Authors

*Email: wangjiangan@nwpu.edu.cn (J. G. Wang); nan1980732@163.com (D. Nan) 


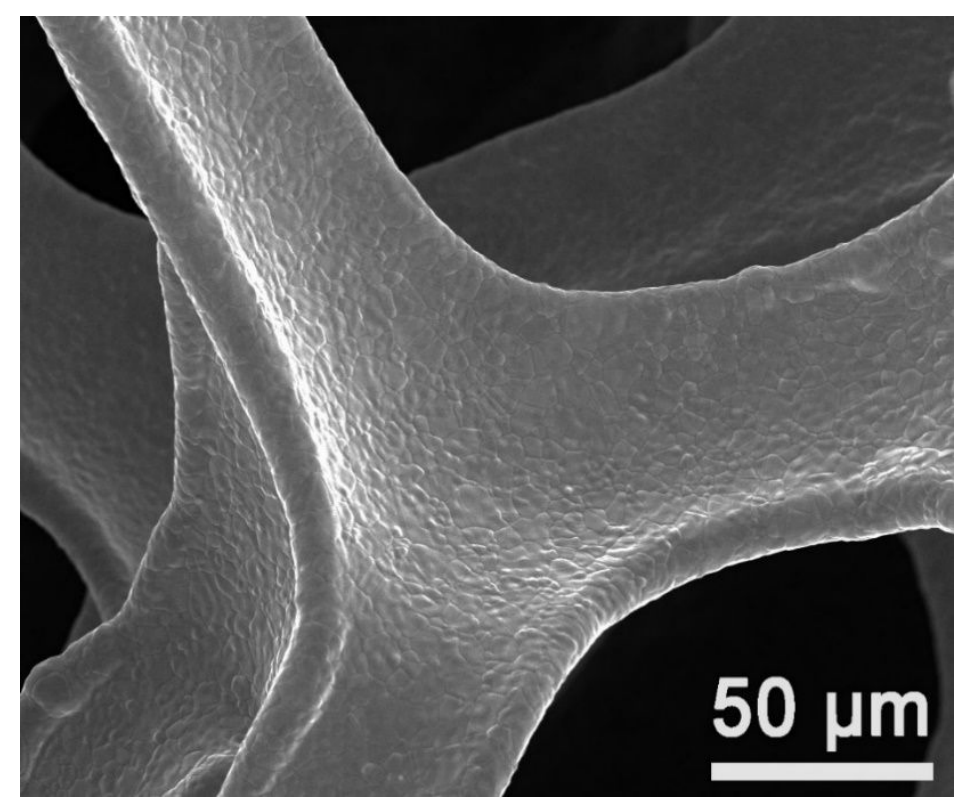

Figure S1. SEM image of the Ni foam. 


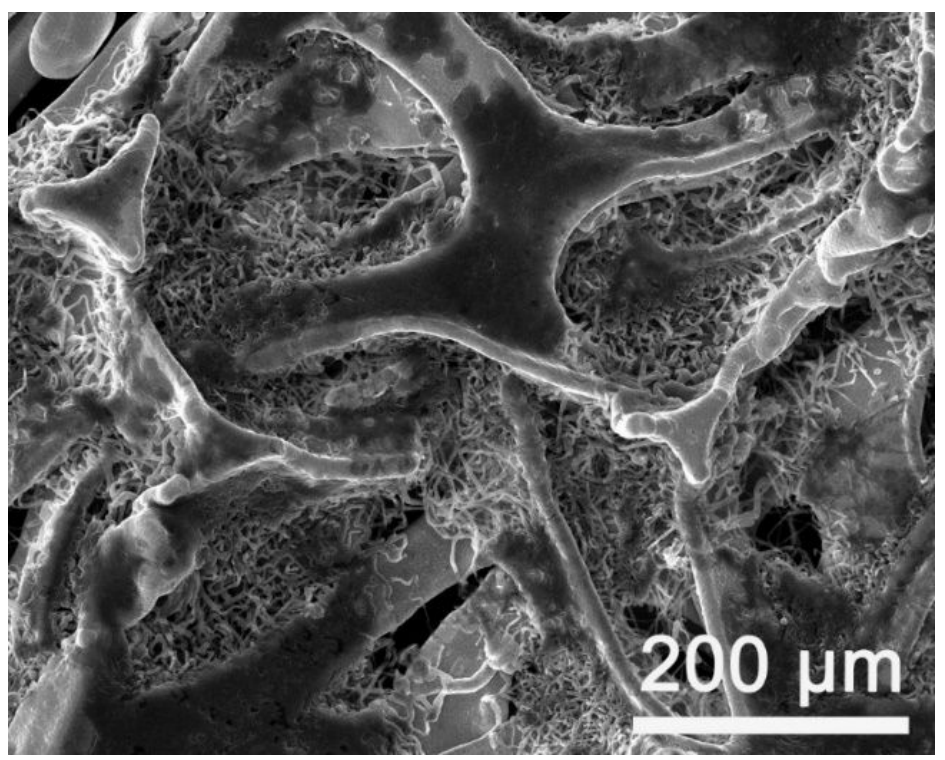

Figure S2. Low resolution image of NF with predepodited $20 \mathrm{mAh} \mathrm{cm}^{-2} \mathrm{Li}$. 


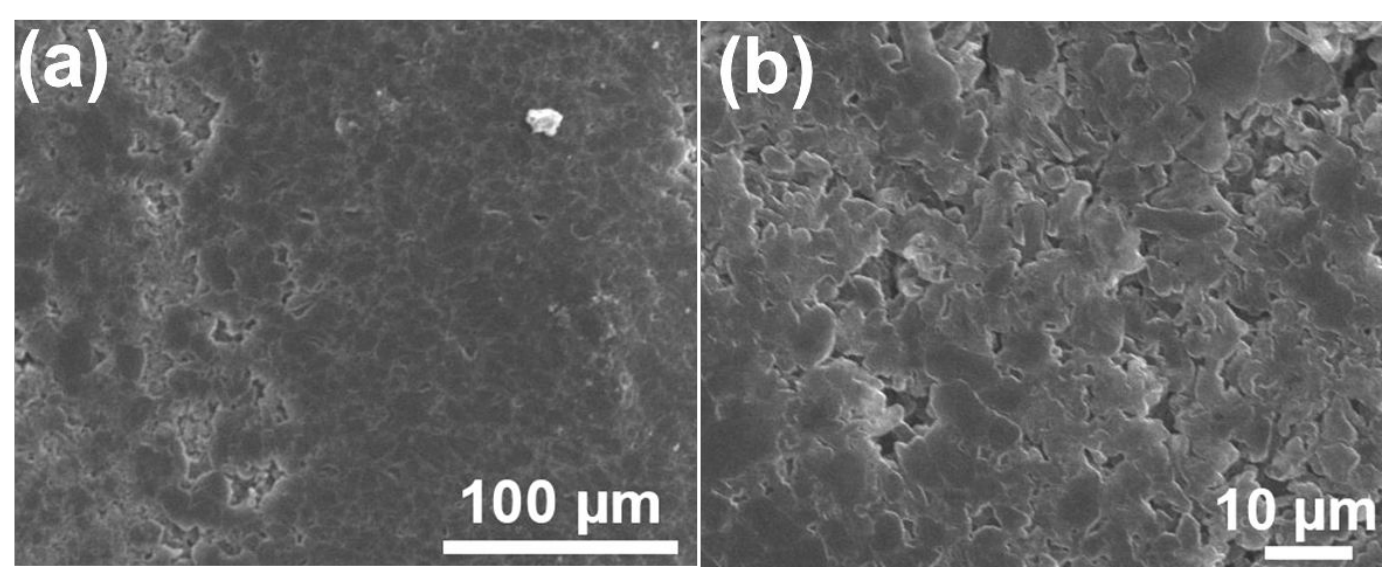

Figure S3. (a) Low and (b) high magnification SEM images of Li@Co 3 N/NF composite electrodes after 500 cycles. 


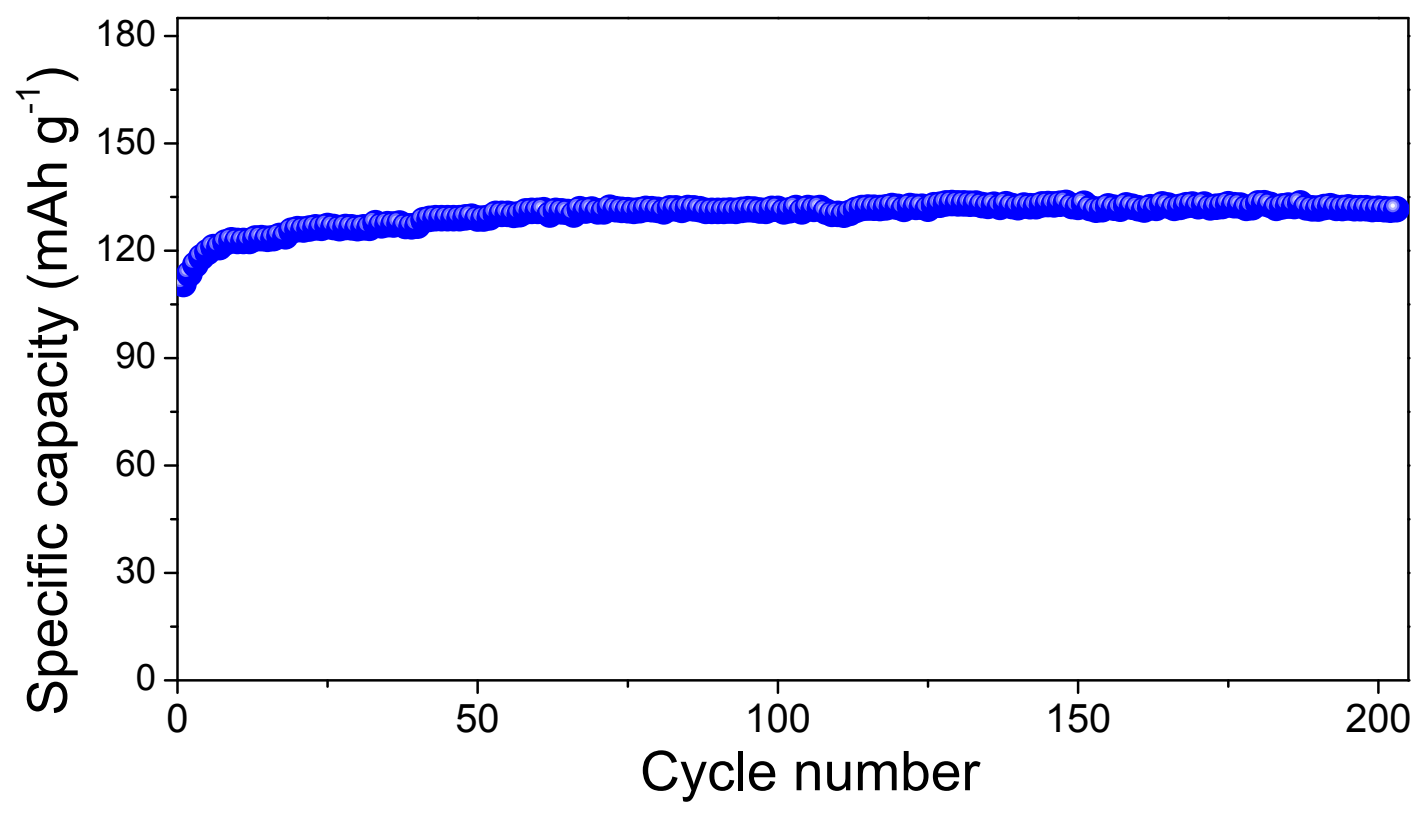

Figure S4. Cycling performance of Li@ $\mathrm{Co}_{3} \mathrm{~N} / \mathrm{NF}-\mathrm{LFP}$ full cell with higher LFP 
loading ( $\left.\sim 5 \mathrm{mg} \mathrm{cm}^{-2}\right)$. 